\title{
Author Correction: Immune dysregulation and immunopathology induced by SARS-CoV-2 and related coronaviruses - are we our own worst enemy?
}

Lok-Yin Roy Wong (i) and Stanley Perlman (i)

Correction to: Nature Reviews Immunology https://doi.org/10.1038/s41577-021-00656-2, published online 26 November 2021.

In Figure 2 in the originally published version of this article, PRD IV and ATF-JUN were incorrectly positioned on the IFN $\beta$ promoter. This error has now been corrected.

https://doi.org/10.1038/s41577-021-00673-1 I Published online 17 December 2021

(๑) Springer Nature Limited 2021 